RAIRO Operations Research

RAIRO Oper. Res. 38 (2004) 105-119

DOI: $10.1051 /$ ro:2004015

\title{
PRODUCT FORM SOLUTION FOR G-NETWORKS WITH DEPENDENT SERVICE
}

\author{
Pavel Bocharov $^{1}$, Ciro D'Apice ${ }^{2}$, Evgeny Gavrilov ${ }^{1}$ \\ AND AleXANDre PECHINKIN ${ }^{3}$
}

\begin{abstract}
We consider a G-network with Poisson flow of positive customers. Each positive customer entering the network is characterized by a set of stochastic parameters: customer route, the length of customer route, customer volume and his service length at each route stage as well. The following node types are considered:

(0) an exponential node with $c_{n}$ servers, infinite buffer and FIFO discipline;

(1) an infinite-server node;

(2) a single-server node with infinite buffer and LIFO PR discipline;

(3) a single-server node with infinite buffer and PS discipline.

Negative customers arriving at each node also form a Poisson flow. A negative customer entering a node with $k$ customers in service, with probability $1 / k$ chooses one of served positive customer as a "target". Then, if the node is of a type 0 the negative customer immediately "kills" (displaces from the network) the target customer, and if the node is of types 1-3 the negative customer with given probability depending on parameters of the target customer route kills this customer and with complementary probability he quits the network with no service. A product form for the stationary probabilities of underlying Markov process is obtained.
\end{abstract}

* This work was supported by the Russian Foundation for Basic Research, Grant No 02-0790147.

1 Department of Probability Theory and Mathematical Statistics Peoples' Friendship University of Russia, Moscow, Russia; e-mail: pbocharov@sci.pfu.edu.ru; tropic_mos@rambler.ru

2 Department of Information Engineering and Applied Mathematics, University of Salerno, Italy; e-mail: dapice@diima.unisa.it

3 Institute of Informatics Problems Russian Academy of Sciences Moscow, Russia;

e-mail: APechinkin@ipiran.ru 


\section{INTRODUCTION}

Queueing networks are widely used for analytical modelling of computer communication networks $[1,2,24,31,34,36]$. A central place in queueing network theory belongs to networks admitting product form of the joint stationary distribution of the number of customers at nodes which we call "multiplicative networks". The theory of multiplicative networks takes its origin from the results of Jackson $[29,30]$ where the solution for an exponential network with nodes of infinite capacity was expressed in a product form for the first time ever. Subsequent weighty contributions to multiplicative network theory were stimulated by the publication formulating the so-called BCMP theorem [3], see also monographs such as $[2,24,25]$, that gives a product solution for a larger class of open networks that are the generalization of the Jackson network. The necessary conditions for a Jackson network to be multiplicative are that all its input flows are Poisson and distributions of customer service times are exponential. For BCMP-networks, the second constraint may not always hold, but then the service disciplines at nodes must be of special types. Subsequent developments in the theory of multiplicative networks resulted from different types of generalizations of the Jackson and BCMP-networks concerned with, for example, the dependence of input flows on the number of customers in a network, dependence of probabilities of transitions between network nodes on the state of these nodes, bypassing of nodes, etc. [1,2,33,35]. A large body of literature exists on approximate methods of decomposition of queueing network models (see, for example, $[1,24,25,32,36]$ ).

An absolutely new class of open networks generalizing the Jackson and BCMPnetworks and admitting product solution was introduced by Gelenbe [10-13], initially motivated by neural networks and associative memory [5]. These networks contain, along with usual (positive) customers, additional Poisson flows of negative customers [8,21]. A negative customer differs from a positive customer in that upon arrival at a network node he kills a positive customer if there is any at this node, thereby reducing the number of positive customers at the node by one. Thereafter the negative customer quits the network, getting no service. Such a network is called the G-network.

The investigation of G-networks has started relatively recently with the first papers of Gelenbe on this topic appearing in 1989. In later publications of Gelenbe and other authors extended most of the results obtained for Jackson and BCMPnetworks and their generalizations to G-networks $[9,14,15,18,20,23,26]$, including batch destruction of normal customers by negative customers, multiple customer classes, and new types of "signals" such as the ability of negative customers to move normal customers to other queues without destroying them, and reset customers. The link with neural networks was also further developed by Gelenbe [16,17,19,22]. Many instances of applications were also presented including [6,7,27,28].

In the present work we give an extension of multiplicative theory for a class of non-traditional BCMP-networks with negative customers. In these extended Gnetworks, a customer is characterized by a set of stochastic parameters: customer route, the length of customer route, customer volume and his service length at each 
route stage as well. Such open networks were analysed in $[4,36]$ where product solution for network nodes of BCMP type was obtained. Thus the present paper generalizes the results of [4] for the case when negative customers are also taken into consideration.

\section{NETWORK DESCRIPTION}

We consider an open queueing network with $M$ nodes and a finite set $\mathcal{M}$ of node types. Each node $s, s=\overline{1, M}$, can be any of the following types:

(0) exponential multi-server with infinite buffer capacity and FCFS discipline (note that the theorem below can be easily extended to exponential nodes with random choice of customers for service from the queue);

(1) infinite-server;

(2) single-server with infinite buffer and LIFO discipline with interruption and resumption;

(3) single-server with infinite buffer and processor sharing (PS) discipline.

We denote by $\mathcal{M}_{i}$ the set of nodes of type $i, i=\overline{0,3}$, and by $c_{s}$ the number of servers in $s$ th (exponential) node.

A Poisson flow of (usual, positive) customers of intensity $\lambda$ enters the network. Each customer arriving at the network is characterized by a set of random variables $(L, \vec{R}, \vec{Y}, \vec{X})$ which depend neither on analogous random variables for other customers nor on network history, where:

- $L$ is a customer route random length, i.e. the number of stages at which he will be served;

- $\vec{R}=\left(R_{1}, \ldots, R_{L}\right)$ is a random route comprising an assembly of node numbers (nodes of the same number at different stages are allowed) the customer subsequently passes at all $L$ stages;

- $\vec{Y}=\left(Y_{1}, \ldots, Y_{L}\right)$ are customer random volumes at route stages the customer subsequently passes (generally speaking, these volumes are different at different stages);

- $\vec{X}=\left(X_{1}, \ldots, X_{L}\right)$ are customer random service lengths at the route stages this customer subsequently passes (service lengths, generally speaking, are different at different stages as well). Note that if the customer is being served at a node of type 2 or 3 , then the service length at each stage coincides with the service time which he would be served if he would stay at that node alone.

The customer volume $\vec{Y}$ can have a real physical meaning as, for instance, a memory volume which is required for message allocation, or it can be interpreted differently. For instance, it can be used to expose customers' types; in this case the network under consideration can be interpreted as a network with continual message set.

It is only natural that under this network description the volume $Y_{n}$ and the length $X_{n}$ define a customer being served at node $R_{n}$. Let us recall that the routes $\vec{R}$ where the numbers $R_{n}$ can be repeated are allowed, i.e. a customer 
can be served at the same node $s$ for the several times but with different service lengths.

Stochastic characteristics of a random variable $(L, \vec{R}, \vec{Y}, \vec{X})$ are given by the joint probability distribution function (PDF)

$$
B(l, \vec{r}, \vec{y}, \vec{x})=\mathbf{P}\left\{L=l, R_{n}=r_{n}, Y_{n} \leq y_{n}, X_{n} \leq x_{n}, \quad n=\overline{1, l}\right\}
$$

Further on, let us denote by

$$
G(l, \vec{r}, \vec{y})=\mathbf{P}\left\{L=l, R_{n}=r_{n}, Y_{n} \leq y_{n}, \quad n=\overline{1, l}\right\}
$$

the joint PDF of the route $\vec{R}$ of the length $L$ and with customer volumes $\vec{Y}$ at the route stages, by

$$
B(\vec{x} \mid l, \vec{r}, \vec{y})=\mathbf{P}\left\{X_{n} \leq x_{n}, \quad n=\overline{1, l} \mid L=l, \vec{R}=\vec{r}, \vec{Y}=\vec{y}\right\}
$$

the conditional joint PDF of the customer service lengths $\vec{X}$ at the route stages under a fixed route $\vec{R}=\vec{r}$ of the length $L=l$ and volumes $\vec{Y}=\vec{y}$, and by

$$
B_{n}(x \mid l, \vec{r}, \vec{y})=\mathbf{P}\left\{X_{n} \leq x \mid L=l, \vec{R}=\vec{r}, \vec{Y}=\vec{y}\right\}, \quad n=\overline{1, l},
$$

the conditional PDF of the customer service length $X_{n}$ at the $n$th stage (at a node with number $R_{n}=r_{n}$ ) under a fixed route $\vec{R}=\vec{r}$ of the length $L=l$ and volumes $\vec{Y}=\vec{y}$.

We shall make the following assumptions on the functions introduced above.

A 1.1. Service lengths are conditionally independent along the route, i.e. the conditional $\operatorname{PDF} B(\vec{x} \mid l, \vec{r}, \vec{y})$ has the form

$$
B(\vec{x} \mid l, \vec{r}, \vec{y})=\prod_{n=1}^{l} B_{n}\left(x_{n} \mid l, \vec{r}, \vec{y}\right) .
$$

A 1.2. Each exponential node $s$ is a $c_{s}$-server queueing system with infinite buffer capacity and service intensity for all customers at each server is equal to $\mu_{s}$. So, if $r_{n}=s \in \mathcal{M}_{0}$, i.e. at an $n$th route stage there is a customer being served at node $s$ of type 0 , then

$$
B_{n}(x \mid l, \vec{r}, \vec{y})=1-\mathrm{e}^{-\mu_{s} x} .
$$

In other words, service length $X_{n}$ at node $R_{n}=s$ of the type 0 depends on neither the route $\vec{R}$, nor any of the the volumes $Y_{k}$ (including the volume $Y_{n}$ ) and has exponential PDF with parameter $\mu_{s}$.

We shall make an additional technical assumptions on the functions introduced above, which could be easily neglected if we interpret derivatives as generalized ones. Namely, we suppose that the PDF $G(l, \vec{r}, \vec{y})$ and $B_{n}(x \mid l, \vec{r}, \vec{y})$ are absolutely continuous, and denote by $g(l, \vec{r}, \vec{y})$ and $b_{n}(x \mid l, \vec{r}, \vec{y})$, respectively, their densities, 
i.e.

$$
g(l, \vec{r}, \vec{y})=\frac{\partial^{l}}{\partial y_{1} \cdots \partial y_{l}} G(l, \vec{r}, \vec{y}), \quad b_{n}(x \mid l, \vec{r}, \vec{y})=\frac{\partial}{\partial x} B_{n}(x \mid l, \vec{r}, \vec{y}) .
$$

Besides of the flow of positive customers described above, negative customers arrive at the network. These flows are defined in the following way.

A 2.1. The flows arriving at different nodes are independent.

A 2.2. A customer flow arriving at node $s$ of type 2 or 3 is Poisson one of intensity $\gamma_{s}$.

A 2.3. A customer flow arriving at node $s$ of either type 0 or 1 is a Markov one with intensity $\gamma_{s}(n)$ depending (only) on the number $n$ of busy servers at this node in the following way: $\gamma_{s}(n)=n \gamma_{s}$.

A 2.4. A negative customer arriving at a node $s$ with $k$ positive customers in service at this node (if this node is of type 0 then $k$ is the number of busy servers, if this node is of the type 1 or 3 , then $k$ is the total number of positive customers at the node, if it is of type 2 , then $k=1$ ), with the same probability $1 / k$ chooses one of positive customers being served. After this, if the node is of the type 0 the negative customer immediately "kills" (displaces from the network) the chosen positive customer. If the node $s$ is of any other type then the negative customer either with probability $\omega_{n}(x \mid l, \vec{r}, \vec{y})$ kills (here and in what follows we shall omit the quotation marks) the chosen positive customer or with the complementary probability $1-\omega_{n}(x \mid l, \vec{r}, \vec{y})$ quits the network without inducing any action. Here $(l, \vec{r}, \vec{y})$ are the parameters of the chosen positive customer, defined earlier: $n$ is the number of route stage in which this customer is served (it is only natural that $r_{n}=s$ ) and $x$ is the elaborated (serviced) customer length. The negative and killed customers quit the network and do not return there ever. If at the moment of a negative customer's arrival into some node there is no positive customer there, then the negative customer quits the network without inducing any action.

\section{Auxiliary functions}

First we introduce for $n=\overline{1, l}$ auxiliary functions $\omega_{n}(l, \vec{r}, \vec{y}), F_{n}(x \mid l, \vec{r}, \vec{y})$ and $B_{n}^{*}(x \mid l, \vec{r}, \vec{y})$, defined for a positive customer with parameters $(l, \vec{r}, \vec{y})$ in the following way (in what follows for any PDF $F(x)$ we shall use the notation $\bar{F}(x)=1-F(x))$

If $r_{n}$ is a node of any of the types $1-3$, then these functions are given by the formulae

$$
\begin{gathered}
\omega_{n}(l, \vec{r}, \vec{y})=\int_{0}^{\infty} \bar{F}_{n}(x \mid l, \vec{r}, \vec{y}) b_{n}(x \mid l, \vec{r}, \vec{y}) \mathrm{d} x, \\
F_{n}(x \mid l, \vec{r}, \vec{y})=1-\exp \left\{-\gamma_{r_{n}} \int_{0}^{x} \omega_{n}(z \mid l, \vec{r}, \vec{y}) \mathrm{d} z\right\}, \\
B_{n}^{*}(x \mid l, \vec{r}, \vec{y})=1-\bar{B}_{n}(x \mid l, \vec{r}, \vec{y}) \bar{F}_{n}(x \mid l, \vec{r}, y) .
\end{gathered}
$$


Similar functions for the node $r_{n}$ of the type 0 have the form

$$
\begin{gathered}
\omega_{n}(l, \vec{r}, \vec{y})=\frac{\mu_{r_{n}}}{\mu_{r_{n}}+\gamma_{r_{n}}}, \\
B_{n}^{*}(x \mid l, \vec{r}, \vec{y})=1-\mathrm{e}^{-\left(\mu_{r_{n}}+\gamma_{r_{n}}\right) x} .
\end{gathered}
$$

Let us set

$$
\begin{aligned}
\omega_{n}^{*}(l, \vec{r}, \vec{y}) & =\prod_{i=1}^{n-1} \omega_{i}(l, \vec{r}, \vec{y}), \quad n=\overline{1, l+1}, \\
g_{n}^{*}(l, \vec{r}, \vec{y}) & =\omega_{n}^{*}(l, \vec{r}, \vec{y}) g(l, \vec{r}, \vec{y}), \quad n=\overline{1, l}, \\
m_{n}(l, \vec{r}, \vec{y}) & =\int_{0}^{\infty} \bar{B}_{n}^{*}(x \mid l, \vec{r}, \vec{y}) \mathrm{d} x, \quad n=\overline{1, l} .
\end{aligned}
$$

As we shall see later these functions have a very transparent physical meaning.

$F_{n}(x \mid l, \vec{r}, \vec{y})$ is the probability that a positive customer with parameters $(l, \vec{r}, \vec{y})$, not killed until the $n$th stage and having an infinite service length at the $n$th stage (at node $r_{n}$ ) will be killed at this stage during the time less than $x$.

$\omega_{n}(l, \vec{r}, \vec{y})$ is the probability that a positive customer with parameters $(l, \vec{r}, \vec{y})$, not killed until the $n$th stage, will not be killed at this stage (at node $r_{n}$ ).

$\omega_{n}^{*}(l, \vec{r}, \vec{y})$ is the probability that a positive customer with parameters $(l, \vec{r}, \vec{y})$ will not be killed until the $n$th stage.

$B_{n}^{*}(x \mid l, \vec{r}, \vec{y})$ is the conditional probability that a positive customer with parameters $(l, \vec{r}, \vec{y})$ will be served (will stay at the server until the moment when either his service is completed or he is killed) at $n$th stage (at node $r_{n}$ ) the time less than $x$ provided that he will not be killed until the $n$th stage.

$m_{n}(l, \vec{r}, \vec{y})$ is the mean service time (sojourn at the server) of a positive customer with parameters $(l, \vec{r}, \vec{y})$ at $n$th stage (at node $r_{n}$ ), not killed until the $n$th stage.

It is only natural that for the nodes of types 2 and 3 the last two characteristics are defined under the condition that there exist no other customers at these nodes.

Let us set for the $s$ th node

$$
\rho_{s}=\lambda \sum_{l=1}^{\infty} \sum_{1 \leq r_{1}, \ldots, r_{l} \leq M} \int_{1} \sum_{n=1}^{l} g_{n}^{*}(l, \vec{r}, \vec{y}) \delta_{s-r_{n}} m_{n}(l, \vec{r}, \vec{y}) \mathrm{d} y
$$

where $\delta_{j}$ is the Kronecker symbol. For the sake of brevity we shall use the notation

$$
\int_{1} \ldots \overrightarrow{\mathrm{d} y}=\int \ldots \int_{1} \mathrm{~d} y_{1} \cdots \mathrm{d} y_{l}
$$

Note that $\rho_{s}$ is the traffic intensity at the $s$ th node. 
Let us suppose that for all nodes $s$

$$
\lambda_{s}=\lambda \sum_{l=1}^{\infty} \sum_{1 \leq r_{1}, \ldots, r_{l} \leq M} \int_{1} \sum_{n=1}^{l} g_{n}^{*}(l, \vec{r}, \vec{y}) \delta_{s-r_{n}} \overrightarrow{d y}<\infty
$$

The last condition means that the total flow intensity $\lambda_{s}$ of positive customers arriving at node $s$ is finite. Note that for non-exponential nodes this condition does not follow from the condition that the traffic intensity of these nodes is finite too (the latter condition will be given below).

And finally, for the sake of brevity we denote by $\beta_{n}^{+}(x \mid l, \vec{r}, \vec{y})$ and $\beta_{n}^{-}(x \mid l, \vec{r}, \vec{y})$, the intensities of departure of positive customer with parameters $(l, \vec{r}, \vec{y})$ from the server at the $n$th route stage (at node $s=r_{n}$ ) owing to the service completion and owing to the killing by a negative customer respectively. These intensities are defined by the formulae:

for nodes of the types $1-3$

$$
\begin{gathered}
\beta_{n}^{+}(x \mid l, \vec{r}, \vec{y})=\frac{b_{n}(x \mid l, \vec{r}, \vec{y})}{\bar{B}_{n}(x \mid l, \vec{r}, \vec{y})}=\frac{b_{n}(x \mid l, \vec{r}, \vec{y})}{\bar{B}_{n}^{*}(x \mid l, \vec{r}, \vec{y})} \bar{F}_{n}(x \mid l, \vec{r}, \vec{y}), \\
\beta_{n}^{-}(x \mid l, \vec{r}, \vec{y})=\gamma_{r_{n}} \omega_{n}(x \mid l, \vec{r}, \vec{y})=\frac{\gamma_{r_{n}} \omega_{n}(x \mid l, \vec{r}, \vec{y})}{\bar{B}_{n}^{*}(x \mid l, \vec{r}, \vec{y})} \bar{B}_{n}(x \mid l, \vec{r}, \vec{y}) \bar{F}_{n}(x \mid l, \vec{r}, \vec{y}),
\end{gathered}
$$

and for nodes of type 0

$$
\begin{aligned}
& \beta_{n}^{+}(x \mid l, \vec{r}, \vec{y})=\mu_{r_{n}}, \\
& \beta_{n}^{-}(x \mid l, \vec{r}, \vec{y})=\gamma_{r_{n}} .
\end{aligned}
$$

\section{MARKOV PROCESS}

Let us now define the Markov process describing the stochastic behaviour of the network under consideration.

We shall denote a network state by an assembly $\vec{z}=\left(\vec{z}_{1}, \ldots, \vec{z}_{M}\right)$, where the assembly $\vec{z}_{s}=\left(k_{s}, \vec{z}_{s 1}, \ldots, \vec{z}_{s k_{s}}\right), s=\overline{1, M}$, in turn, describes the state of the $s$ th node in the following way: $k_{s}$ is the number of customer at the $s$ th node and the assembly $\vec{z}_{s i}, \quad s=\overline{1, M}, \quad i=\overline{1, k_{s}}$, with components $\vec{z}_{s i}=\left(l_{s i}, \vec{r}_{s i}, \vec{y}_{s i}, n_{s i}, x_{s i}\right)$ stores the information $\left(l_{s i}, \vec{r}_{s i}, \vec{y}_{s i}\right)$ on the $i$ th customer at the $s$ th node, and his position $\left(n_{s i}, x_{s i}\right)$ in the network:

$-l_{s i}$ is the route length;

$-\vec{r}_{s i}=\left(r_{s i 1}, \ldots, r_{s i l_{s i}}\right)$ is the route;

- $\vec{y}_{s i}=\left(y_{s i 1}, \ldots, y_{s i l_{s i}}\right)$ are customer volumes at route stages;

- $n_{s i}$ is the route stage number, the customer is passing (while being serviced or waiting for service); clearly that $n_{s i} \leq l_{s i}$;

- $x_{s i}$ is the customer length already serviced at a given stage. 
Evidently that due to the notations introduced above, we have $r_{s i n_{s i}}=s$. It is also clear that the vector $\vec{z}_{s}=0$ if $k_{s}=0$, i.e. when there are no customers at the $s$ th node, and the vector $\vec{z}=\mathbf{0}=(0, \ldots, 0)$ in the case, when there are no customers in the network at all. Moreover we shall consider that the coordinates $x_{s i}$ are not defined for exponential nodes, i.e. when $s \in \mathcal{M}_{0}$ (the length already serviced at this stage is not taken into consideration), although, like as before, for the sake of uniformity we shall preserve the argument $x_{s i}$.

In what follows we will accept the following rule of numbering of customers in the nodes. For the nodes of type 0 the numbers are assigned to the customers in the order of their arrivals at the nodes, for the nodes of types 1 or 3 , the numbers are assigned randomly, and for the nodes of type 2 , in the inverse order to their arrivals at the nodes.

We denote by $\mathcal{Z}=\{\vec{z}\}$ the state space of the network. The behaviour of the network can be described by a Markov process $\{\vec{z}(t), t \geq 0\}$ with the state space $\mathcal{Z}$. So $\vec{Z}(t)=\vec{z}$, if at the instant $t$ the network is in the state $\vec{z}$.

\section{Product FORM SOLUtion}

In this section we will prove the theorem on the multiplicative representation of the stationary probabilities of states for the Markov process under consideration.

The stationary probability density of states of the process $\vec{z}(t)$ will be denoted by $p(\vec{z})$. In the text below, we shall show in an explicit way the existence of this probability density under the natural constraints on the network traffic imposed above.

Theorem 5.1. If for a node $s$ of type $0 \rho_{s}<c_{s}$, for a node $s$ of type $1 \rho_{s}<\infty$, and for a node $s$ of types 2 and $3 \rho_{s}<1$, where $\rho_{s}$ is defined by the formula (7), then there exists a limit (stationary) probability state distribution of the process $\vec{z}(t)$ with probability distribution density

$$
p(\vec{z})=\prod_{s=1}^{M} p_{s}\left(\vec{z}_{s}\right)
$$

thereby:

for a node $s$ of the type 0

$$
p_{s}\left(\vec{z}_{s}\right)=p_{s}(0) d_{s}\left(k_{s}\right)\left(\frac{\lambda}{\mu_{s}+\gamma_{s}}\right)^{k_{s}} \prod_{i=1}^{k_{s}} g_{n_{s i}}^{*}\left(l_{s i}, \vec{r}_{s i}, \vec{y}_{s i}\right)
$$

where

$$
p_{s}(0)=\left(\sum_{i=0}^{c_{s}} \frac{\rho_{s}^{i}}{i !}+\frac{\rho_{s}^{c_{s}+1}}{c_{s} !\left(c_{s}-\rho_{s}\right)}\right)^{-1},
$$




$$
d_{s}\left(k_{s}\right)= \begin{cases}1 / k_{s} ! & \text { if } k_{s} \leq c_{s} \\ 1 /\left(c_{s} ! c_{s}^{k_{s}-c_{s}}\right) & \text { if } k_{s}>c_{s}\end{cases}
$$

for a node $s$ of the type 1

$$
p_{s}\left(\vec{z}_{s}\right)=\mathrm{e}^{-\rho_{s}} \frac{\lambda^{k_{s}}}{k_{s} !} \prod_{i=1}^{k_{s}} \bar{B}_{n_{s i}}^{*}\left(x_{s i} \mid l_{s i}, \vec{r}_{s i}, \vec{y}_{s i}\right) g_{n_{s i}}^{*}\left(l_{s i}, \vec{r}_{s i}, \vec{y}_{s i}\right)
$$

for a node $s$ of the type 2 or 3

$$
p_{s}\left(\vec{z}_{s}\right)=\left(1-\rho_{s}\right) \lambda^{k_{s}} \prod_{i=1}^{k_{s}} \bar{B}_{n_{s i}}^{*}\left(x_{s i} \mid l_{s i}, \vec{r}_{s i}, \vec{y}_{s i}\right) g_{n_{s i}}^{*}\left(l_{s i}, \vec{r}_{s i}, \vec{y}_{s i}\right) .
$$

Proof. It can be easily seen that the Markov process $\vec{z}(t)$ is regular and Harris positively recurrent because the state $\mathbf{0}$ is a positively recurrent atom for this process. Therefore to prove the theorem it is sufficient to show that the function $p(\vec{z})$, defined by theorem assertions satisfies the system of equations for the stationary density of states of the process $\vec{z}(t)$, which we shall call the system of equilibrium equations by analogy with the discrete case.

For the system of equilibrium equations we introduce the following notations. Let the network be in the state $\vec{z}$. Then $v_{s i}(\vec{z})$ identifies the service rate of the $i$ th customer staying in the node $s$, i.e.

$$
v_{s i}(\vec{z})=
$$$$
\begin{cases}1, & \begin{array}{l}
\text { if the } i \text { th customer is served in a node } s \text { of the type } 1 \\
\text { or if the } i \text { th customer engages the server in a node } s \text { of type } 0 \text { or } 2 ;
\end{array} \\
1 / k_{s}, & \begin{array}{l}
\text { if the } i \text { th customer is served in the node } s \text { of the type } 3 \\
\text { (in which other } k_{s}-1 \text { customers are also being served); }
\end{array} \\
0, & \text { if the } i \text { th customer remains in the queue at the node } s \text { of type } 0 \text { or } 2,\end{cases}
$$

and

and

$$
\mu_{s i}^{+}(\vec{z})=v_{s i}(\vec{z}) \beta_{n_{s i}}^{+}\left(x_{s i} \mid l_{s i}, \vec{r}_{s i}, \vec{y}_{s i}\right)
$$

$$
\mu_{s i}^{-}(\vec{z})=v_{s i}(\vec{z}) \beta_{n_{s i}}^{-}\left(x_{s i} \mid l_{s i}, \vec{r}_{s i}, \vec{y}_{s i}\right)
$$

are the output intensities from the state $\vec{z}$ owing, respectively, to the service completion or killing of an $i$ th customer at the node $s$.

Moreover, for $s \in \mathcal{M}_{1} \cup \mathcal{M}_{2} \cup \mathcal{M}_{3}, k>0$, and $i=\overline{1, k}$ let us set

$$
u_{s i}(k)= \begin{cases}1, & \text { if } s \in \mathcal{M}_{2} \text { and } i=1 ; \\ 0, & \text { if } s \in \mathcal{M}_{2} \text { and } i>1 ; \\ 1 / k, & \text { if } s \in \mathcal{M}_{1} \cup \mathcal{M}_{3} .\end{cases}
$$


The function $u_{s i}(k)$ is the probability that to a customer, arriving at a nonexponential node $s$ with $k-1$ customers also staying there, is assigned a number $i$.

Since for different states of the set $\mathcal{Z}$ of the process $\vec{Z}(t)$, the equilibrium equations will be different we divide the set $\mathcal{Z}$ into two subsets of states. We assign all states with $x_{s i}>0$ for all $(s, i)$ they are defined (i.e. for all $\left.s \in \mathcal{M} \backslash \mathcal{M}_{0}\right)$ to the first subset. Such states will be called inner states. It is obvious that an inner state is a state in which all the customers residing in the nodes of the types 1-3 have already received service for at least some time. The remaining states will be assigned to the second subset and called boundary states.

Let us start with inner states, and assign each inner state $\vec{z} \in \mathcal{Z}$ a set of states $\tilde{\mathcal{Z}}(\vec{z})$ to. The states from of the set $\tilde{\mathcal{Z}}(\vec{z})$ will be called the states preceding the state $\vec{z}$. The reason why we introduce the preceding states is that the direct transition into the state $\vec{z}$ is only possible from these states.

In turn, all states preceding an inner state $\vec{z}$, will be divided into 4 classes. Each class will be associated to a certain transition into the state $\vec{z}$.

The first class $\mathcal{Z}^{+}(\vec{z})=\mathcal{Z}_{1}^{+}(\vec{z}) \cup \mathcal{Z}_{2}^{+}(\vec{z})$ consists of two disjoint subclasses $\mathcal{Z}_{1}^{+}(\vec{z})$ and $\mathcal{Z}_{2}^{+}(\vec{z})$.

The first subclass $\mathcal{Z}_{1}^{+}(\vec{z})=\left\{\vec{z}^{+}(\vec{z}, s, i, l, \vec{r}, \vec{y}, x)\right\}$, is a set of network states from which a transition to the state $\vec{z}$ is possible owing to the exit of a customer from the network after the completion of his service (at the route's last node) and contains only the states $\vec{z}^{+}(\vec{z}, s, i, l, \vec{r}, \vec{y}, x)$ of the form

$$
\vec{z}^{+}(\vec{z}, s, i, l, \vec{r}, \vec{y}, x)=\left(\vec{z}_{1}, \ldots, \vec{z}_{s-1}, \vec{z}_{s}^{*}, \vec{z}_{s+1}, \ldots, \vec{z}_{M}\right),
$$

where $\vec{z}_{s}^{*}=\left(k_{s}+1, \vec{z}_{s 1}^{*}, \ldots, \vec{z}_{s, k_{s}+1}^{*}\right)$ and

$$
\vec{z}_{s j}^{*}= \begin{cases}\vec{z}_{s j}, & j<i, \\ (l, \vec{r}, \vec{y}, l, x), & j=i, \\ \vec{z}_{s, j-1}, & j>i,\end{cases}
$$

and $(l, \vec{r}, \vec{y}, x)$ are the parameters of the completely served customer which departs from the $i$ th place at the $s$ th node, which can take any (possible) values.

The second subclass $\mathcal{Z}^{+}(\vec{z})=\left\{\vec{z}^{+}(\vec{z}, s, i, \vec{r}, \vec{y}, x)\right\}$, is a set of network states from which a transition to the state $\vec{z}$ is possible owing to the exit of a customer from the network after his killing at the $i$ th place at the $n$th route's stage node and contains only the states $\vec{z}^{+}(\vec{z}, s, i, l, \vec{r}, \vec{y}, n, x)$ of the form

$$
\vec{z}^{+}(\vec{z}, s, i, l, \vec{r}, \vec{y}, n, x)=\left(\vec{z}_{1}, \ldots, \vec{z}_{s-1}, \vec{z}_{s}^{*}, \vec{z}_{s+1}, \ldots, \vec{z}_{M}\right),
$$

where $\vec{z}_{s}^{*}=\left(k_{s}+1, \vec{z}_{s 1}^{*}, \ldots, \vec{z}_{s, k_{s}+1}^{*}\right)$ and

$$
\vec{z}_{s j}^{*}= \begin{cases}\vec{z}_{s j}, & j<i, \\ (l, \vec{r}, \vec{y}, n, x), & j=i, \\ \vec{z}_{s, j-1}, & j>i,\end{cases}
$$

and parameters $(l, \vec{r}, \vec{y}, n, x)$ can take any possible values. 
The remaining 3 classes of preceding states are not empty only for the networks which have any non-exponential nodes.

Let $s \in \mathcal{M}_{0}$ and $\vec{z}$ be such a state that $k_{s}>0$. We also suppose that $n_{s k_{s}}=1$. Let us denote by $\vec{z}^{-}(\vec{z}, s)$ the state

$$
\vec{z}^{-}(\vec{z}, s)=\left(\vec{z}_{1}, \ldots, \vec{z}_{s-1}, \vec{z}_{s}^{*}, \vec{z}_{s+1}, \ldots, \vec{z}_{M}\right)
$$

where $\vec{z}_{s}^{*}=\left(k_{s}-1, \vec{z}_{s 1}, \ldots, \vec{z}_{s, k_{s}-1}\right)$. The states of the set $\mathcal{Z}_{1}^{-}(\vec{z})$ will be called the states preceding the state $\vec{z}$. We also denote the set of such nodes $s$ for which the states $\vec{z}^{-}(\vec{z}, s)$ are defined by $\mathcal{S}_{1}(\vec{z})$. Evidently, the class $\mathcal{Z}_{1}^{-}(\vec{z})$ contains the states from which a transition into the state $\vec{z}$ is possible owing to the arrival of a new customer at an exponential network node, and the set $\mathcal{S}_{1}(\vec{z})$ contains exponential nodes in which, while the network is in state $\vec{z}$, a customer just arrived at the network is placed into the last position in the queue.

The class $\mathcal{Z}_{2}^{-}(\vec{z})$ is associated to the preceding states from which a transition into the state $\vec{z}$ is performed owing to the service completion of a customer in an exponential node and his arrival to another exponential node (at the last place), and contains disjoint subclasses $\mathcal{Z}_{2}^{-}(\vec{z}, s)$ defined in the following way. Let $s \in$ $\mathcal{M}_{0}$ and $\vec{z}$ be such a state that $k_{s}>0$. We also suppose that $n_{s k_{s}}>1$ and

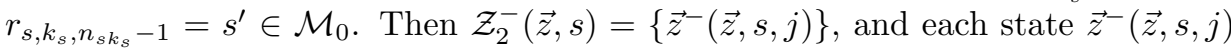
has the form

$$
\overrightarrow{z^{-}}(\vec{z}, s, j)=\left(\vec{z}_{1}, \ldots, \vec{z}_{s^{\prime}-1}, \vec{z}_{s^{\prime}}, \vec{z}_{s^{\prime}+1}, \ldots, \vec{z}_{s-1}, \vec{z}_{s}^{*}, \vec{z}_{s+1}, \ldots, \vec{z}_{M}\right)
$$

(it is only natural that $s$ can precede to $s^{\prime}$ and even coincide with $s^{\prime}$, if a customer from the $s$ th node arrives again at the $s$ th node), where

$$
\begin{gathered}
\vec{z}_{s^{\prime}}^{*}=\left(k_{s^{\prime}}+1, \vec{z}_{s^{\prime} 1}^{*}, \ldots, \vec{z}_{s^{\prime}, k_{s^{\prime}}+1}^{*}\right), \\
\vec{z}_{s^{\prime} i}^{*}= \begin{cases}\vec{z}_{s^{\prime} i}, & i<j, \\
\left(l_{s k_{s}}, \vec{r}_{s k_{s}}, \vec{y}_{s k_{s}}, n_{s k_{s}}-1, x\right), & i=j, \\
\vec{z}_{s^{\prime}, i-1}, & i>j,\end{cases} \\
\vec{z}_{s}^{*}=\left(k_{s}-1, \vec{z}_{s 1}, \ldots, \vec{z}_{s, k_{s}-1}\right) .
\end{gathered}
$$

We shall denote by $\mathcal{S}_{2}(\vec{z})$ the set of nodes for which subclasses $\mathcal{Z}_{2}^{-}(\vec{z}, s)$ are nonempty.

And finally, the class $\mathcal{Z}_{3}^{-}(\vec{z})$ is analogous to the class $\mathcal{Z}_{2}^{-}(\vec{z})$ except the case when a transition into the state $\vec{z}$ is performed owing to a service completion at a non-exponential node. Disjoint classes $\mathcal{Z}_{3}^{-}(\vec{z}, s)$, forming the class $\mathcal{Z}_{3}^{-}(\vec{z})$, are defined as follows. Let $s \in \mathcal{M}_{0}$ and $\vec{z}$ be such a state that $k_{s}>0$. We also suppose $n_{s k_{s}}>1$ and $r_{s, k_{s}, n_{s k_{s}}-1}=s^{\prime} \notin \mathcal{M}_{0}$. Then $\mathcal{Z}_{3}^{-}(\vec{z}, s)=\left\{\vec{z}^{-}(\vec{z}, s, j, x)\right\}$, and each state $\vec{z}^{-}(\vec{z}, s, j, x)$ has the form

$$
\vec{z}^{-}(\vec{z}, s, j, x)=\left(\vec{z}_{1}, \ldots, \vec{z}_{s^{\prime}-1}, \vec{z}_{s^{\prime}}^{*}, \vec{z}_{s^{\prime}+1}, \ldots, \vec{z}_{s-1}, \vec{z}_{s}^{*}, \vec{z}_{s+1}, \ldots, \vec{z}_{M}\right),
$$


where

$$
\begin{gathered}
\vec{z}_{s^{\prime}}^{*}=\left(k_{s^{\prime}}+1, \vec{z}_{s^{\prime} 1}^{*}, \ldots, \vec{z}_{s^{\prime}, k_{s^{\prime}}+1}^{*}\right), \\
\vec{z}_{s^{\prime} i}^{*}= \begin{cases}\vec{z}_{s^{\prime} i}, & i<j, \\
\left(l_{s k_{s}}, \vec{r}_{s k_{s}}, \vec{y}_{s k_{s}}, n_{s k_{s}}-1, x\right), & i=j, \\
\vec{z}_{s^{\prime}, i-1}, & i>j,\end{cases} \\
\vec{z}_{s}^{*}=\left(k_{s}-1, \vec{z}_{s 1}, \ldots, \vec{z}_{s, k_{s}-1}\right) .
\end{gathered}
$$

We shall denote by $\mathcal{S}_{3}(\vec{z})$ the set of nodes $s$, for which the subclasses $\mathcal{Z}_{3}^{-}(\vec{z}, s)$ are non-empty.

Note once again that each of the classes $\mathcal{Z}_{1}^{-}(\vec{z})-\mathcal{Z}_{3}^{-}(\vec{z})$ can be empty. In particular, for the state $\mathbf{0}=(0, \ldots, 0)$ all classes $\mathcal{Z}_{1}^{-}(\vec{z})-\mathcal{Z}_{3}^{-}(\vec{z})$ are empty.

Now we shall write the system of equilibrium equations for inner states $\vec{z}$ :

$$
\begin{aligned}
& \sum_{s \notin \mathcal{M}_{0}} \sum_{i=1}^{k_{s}} \frac{\partial}{\partial x_{s i}}\left(v_{s i}(\vec{z}) p(\vec{z})\right)+\left(\lambda+\sum_{s=1}^{M} \sum_{i=1}^{k_{s}}\left(\mu_{s i}^{+}(\vec{z})+\mu_{s i}^{-}(\vec{z})\right)\right) p(\vec{z}) \\
& \quad=\sum_{s \in \mathcal{M}_{0}} \sum_{i=1}^{k_{s}+1} \sum_{l, \vec{m} r} \int_{1} \mu_{s i}^{+}\left(\vec{z}^{+}(\vec{z}, s, i, l, \vec{r}, \vec{y}, x)\right) p\left(\vec{z}^{+}(\vec{z}, s, i, l, \vec{r}, \vec{y}, x)\right) \mathrm{d} y \\
& +\sum_{s \notin \mathcal{M}_{0}} \sum_{i=1}^{k_{s}+1} \sum_{l, \vec{m} r} \int_{1} \overrightarrow{\mathrm{d} y} \int_{0}^{\infty} \mu_{s i}^{+}\left(\vec{z}^{+}(\vec{z}, s, i, l, \vec{r}, \vec{y}, x)\right) p\left(\vec{z}^{+}(\vec{z}, s, i, l, \vec{r}, \vec{y}, x)\right) \mathrm{d} x \\
& +\sum_{s \in \mathcal{M}_{0}} \sum_{i=1}^{k_{s}+1} \sum_{l, \vec{m} r, n} \int_{1} \mu_{s i}^{-}\left(\vec{z}^{+}(\vec{z}, s, i, l, \vec{r}, \vec{y}, n, x)\right) p\left(\vec{z}^{+}(\vec{z}, s, i, l, \vec{r}, \vec{y}, n, x)\right) \mathrm{d} y \\
& +\sum_{s \notin \mathcal{M}_{0}} \sum_{i=1}^{k_{s}+1} \sum_{l, \vec{m} r, n} \int_{1} \overrightarrow{\mathrm{d} y} \int_{0}^{\infty} \mu_{s i}^{-}\left(\vec{z}^{+}(\vec{z}, s, i, l, \vec{r}, \vec{y}, n, x)\right) p\left(\vec{z}^{+}(\vec{z}, s, i, l, \vec{r}, \vec{y}, n, x)\right) \mathrm{d} x \\
& +\sum_{s \in \mathcal{S}_{1}(\vec{z})} \lambda p(\vec{z}(\vec{z}, s)) g\left(l_{s k_{s}}, \vec{r}_{s k_{s}}, \vec{y}_{s k_{s}}\right)+\sum_{s \in \mathcal{S}_{2}(\vec{z})} \sum_{j=1}^{k_{s^{\prime}}+1} \mu_{s^{\prime} j}^{+}(\vec{z}(\vec{z}, s, j)) p(\vec{z}(\vec{z}, s, j)) \\
& +\sum_{s \in \mathcal{S}_{3}(\vec{z})} \sum_{j=1}^{k_{s^{\prime}+1}} \int_{0}^{\infty} \mu_{s^{\prime} j}^{+}(\vec{z}-(\vec{z}, s, j, x)) p(\vec{z}(\vec{z}, s, j, x)) \mathrm{d} x . \quad(23)
\end{aligned}
$$

We now pass on to the boundary states. We shall limit ourselves to consideration of boundary states $\vec{z}$ for which $x_{s i}=0$ only for a pair $(s, i)$. The case when some elaborated service lengths are equal to zero simultaneously can be studied in a similar way. Moreover for nodes $s$ of the type 3 we consider only such states for which $x_{s 1}=0$, because for nodes of these types in the case $x_{s i}=0, i>1$, the system of equilibrium equations is composed in the same way as for inner states. 
Let $\vec{z}$ be a boundary state, i.e. such a state that $x_{s i}=0$ for some $s \in \mathcal{M} \backslash \mathcal{M}_{0}$ and $i$. We assign each state to one of 3 types in accordance with the following rule.

Let $n_{s i}=1$. Then a state $\vec{z}$ is associated to the first type of boundary states. It is clear that a transition to a boundary state $\vec{z}$ of the first type is realized owing to a new customer arrival at the network, where he is placed at the $i$ th position at an $s$ th (non-exponential) node.

If $n_{s i}>1$ and $r_{s, i, n_{s i}-1}=s^{\prime} \in \mathcal{M}_{0}$, then a state $\vec{z}$ is associated to the second type of boundary states. A transition to a boundary state $\vec{z}$ of the second type is realized owing to the arrival at the $i$ th place at an $s$ th (non-exponential) node of customer which has been served earlier at an exponential node.

Finally, if $n_{s i}>1$ and $r_{s, i, n_{s i}-1}=s^{\prime} \in \mathcal{M} \backslash \mathcal{M}_{0}$ a state $\vec{z}$ is associated to the third type of boundary states. A transition to a boundary state $\vec{z}$ of the third type is realized owing to the arrival at the $i$ th place in $s$ th (non-exponential) node of a customer who has been served earlier at a non-exponential node.

As it has been made earlier for an inner state, for a boundary state $\vec{z}$ we define a set $\tilde{\mathcal{Z}}(\vec{z})$ of preceding states.

For a boundary state $\vec{z}$ of the first type the set $\tilde{\mathcal{Z}}(\vec{z})$ contains only one state

$$
\vec{z}^{-}(\vec{z})=\left(\vec{z}_{1}, \ldots, \vec{z}_{s-1}, \vec{z}_{s}^{*}, \vec{z}_{s+1}, \ldots, \vec{z}_{m}\right),
$$

where

$$
\vec{z}_{s}^{*}=\left(k_{s}-1, \vec{z}_{s 1}, \ldots, \vec{z}_{s, i-1}, \vec{z}_{s, i+1}, \ldots, \vec{z}_{s, k_{s}}\right) .
$$

For a boundary state $\vec{z}$ of the second type the set $\tilde{\mathcal{Z}}(\vec{z})$ contains states $\vec{z}^{-}(\vec{z}, j)$ of the type

$$
\vec{z}^{-}(\vec{z}, j)=\left(\vec{z}_{1}, \ldots, \vec{z}_{s^{\prime}-1}, \vec{z}_{s^{\prime}}^{*}, \vec{z}_{s^{\prime}+1}, \ldots, \vec{z}_{s-1}, \vec{z}_{s}^{*}, \vec{z}_{s+1}, \ldots, \vec{z}_{m}\right),
$$

where

$$
\begin{gathered}
\vec{z}_{s^{\prime}}^{*}=\left(k_{s^{\prime}}+1, \vec{z}_{s^{\prime} 1}^{*}, \ldots, \vec{z}_{s^{\prime}, k_{s^{\prime}}+1}^{*}\right), \\
\vec{z}_{s^{\prime} t}^{*}= \begin{cases}\vec{z}_{s^{\prime} t}, & t<j, \\
\left(l_{s i}, \vec{r}_{s i}, \vec{y}_{s i}, n_{s i}-1, x\right), & t=j, \\
\vec{z}_{s^{\prime}, t-1}, & t>j,\end{cases} \\
\vec{z}_{s}^{*}=\left(k_{s}-1, \vec{z}_{s 1}, \ldots, \vec{z}_{s, i-1}, \vec{z}_{s, i+1}, \ldots, \vec{z}_{s, k_{s}}\right) .
\end{gathered}
$$

For a boundary state $\vec{z}$ of the third type the set $\tilde{\mathcal{Z}}(\vec{z})$ contains states $\vec{z}^{-}(\vec{z}, j, x)$ of the type

$$
\vec{z}^{-}(\vec{z}, j, x)=\left(\vec{z}_{1}, \ldots, \vec{z}_{s^{\prime}-1}, \vec{z}_{s^{\prime}}^{*}, \vec{z}_{s^{\prime}+1}, \ldots, \vec{z}_{s-1}, \vec{z}_{s}^{*}, \vec{z}_{s+1}, \ldots, \vec{z}_{m}\right),
$$

where

$$
\vec{z}_{s^{\prime}}^{*}=\left(k_{s^{\prime}}+1, \vec{z}_{s^{\prime} 1}^{*}, \ldots, \vec{z}_{s^{\prime}, k_{s^{\prime}}+1}^{*}\right),
$$




$$
\begin{gathered}
\vec{z}_{s^{\prime} t}^{*}= \begin{cases}\vec{z}_{s^{\prime} t}, & t<j, \\
\left(l_{s i}, \vec{r}_{s i}, \vec{y}_{s i}, n_{s i}-1, x\right), & t=j, \\
\vec{z}_{s^{\prime}, t-1}, & t>j,\end{cases} \\
\vec{z}_{s}^{*}=\left(k_{s}-1, \vec{z}_{s 1}, \ldots, \vec{z}_{s, i-1}, \vec{z}_{s, i+1}, \ldots, \vec{z}_{s, k_{s}}\right) .
\end{gathered}
$$

We now shall write the equilibrium equations for the boundary states $\vec{z}$ of different types for which $x_{s i}=0$ :

$$
\begin{gathered}
v_{s i}(\vec{z}) p(\vec{z})=\lambda p\left(\vec{z}^{-}(\vec{z})\right) g\left(l_{s i}, \vec{r}_{s i}, \vec{y}_{s i}\right) u_{s i}\left(k_{s}\right), \\
v_{s i}(\vec{z}) p(\vec{z})=\sum_{j=1}^{k_{s^{\prime}}+1} \mu_{s^{\prime} j}^{+}(\vec{z}(\vec{z}, j)) p\left(\vec{z}^{-}(\vec{z}, j)\right) u_{s i}\left(k_{s}\right), \\
v_{s i}(\vec{z}) p(\vec{z})=\sum_{j=1}^{k_{s^{\prime}+1}} u_{s i}\left(k_{s}\right) \int_{0}^{\infty} \mu_{s^{\prime} j}^{+}\left(\vec{z}^{-}(\vec{z}, j, x)\right) p(\vec{z}(\vec{z}, j, x)) \mathrm{d} x .
\end{gathered}
$$

It can be shown that a direct substitution of formulae (13)-(18) into the systems of equations (23) and (24) leads to systems of identities. The proof of theorem is completed by verification of the fact that the function $p(\vec{z})$ satisfies the normalization condition.

Acknowledgement. Authors are very grateful to Professor E. Gelenbe who offered useful comments and valuable suggestions for improvement of the present work.

\section{REFERENCES}

[1] G.P. Basharin, P.P. Bocharov and Ya. A. Kogan, Analysis of Queues in Computer Networks. Theory and Design Methods, Moscow, Nauka (1989) (in Russian).

[2] G.P. Basharin and A.L. Tolmachev, The theory of queueing networks and its application to analysis of information-computer systems, in Itogi Nauki i Tekhniki. Teoria Veroyatnostei, Mat. Statistika, Teoret. Kibertetika 21 3-120. Moscow, VINITI (1983).

[3] F. Baskett, K.M. Chandy, R.R. Muntz and F.G. Palacios, Open, closed and mixed networks of queues with different classes of customers. J. ACM 22 (1975) 248-260.

[4] P.P. Bocharov and V.M. Vishnevskii, G-networks: development of the theory of multiplicative networks. Autom. Remote Control 64 (2003) 714-739.

[5] J. Bourrely and E. Gelenbe, Mémoires associatives: évaluation et architectures. C.R. Acad. Sci. Paris II 309 (1989) 523-526.

[6] C. Cramer and E. Gelenbe, Video quality and traffic QoS in learning-based subsampled and receiver-interpolated video sequences. IEEE J. on Selected Areas in Communications 18 (2000) 150-167.

[7] Y. Feng and E. Gelenbe, Adaptive object tracking and video compression. Network and Information Systems J. 1 (1999) 371-400.

[8] E. Gelenbe, Queuing networks with negative and positive customers. J. Appl. Prob. 28 (1991) 656-663.

[9] J.-M. Fourneau, E. Gelenbe and R. Suros, G-networks with multiple classes of positive and negative customers. Theoret. Comp. Sci. 155 (1996) 141-156.

[10] E. Gelenbe, Réseaux stochastiques ouverts avec clients négatifs et positifs, et réseaux neuronaux. C.R. Acad. Sci. Paris II 309 (1989) 979-982. 
[11] E. Gelenbe, Random neural networks with positive and negative signals and product form solution. Neural Comput. 1 (1989) 502-510.

[12] E. Gelenbe, Réseaux neuronaux aléatoires stables. C.R. Acad. Sci. II 310 (1990) 177-180.

[13] E. Gelenbe, Stable random neural networks. Neural Comput. 2 (1990) 239-247.

[14] E. Gelenbe, G-networks with instantaneous customer movement. J. Appl. Probab. 30 (1993) $742-748$.

[15] E. Gelenbe, G-Networks with signals and batch removal. Probab. Eng. Inform. Sci. 7 (1993) 335-342.

[16] E. Gelenbe, Learning in the recurrent random network. Neural Comput. 5 (1993) 154-164.

[17] E. Gelenbe, G-networks: An unifying model for queuing networks and neural networks. Ann. Oper. Res. 48 (1994) 433-461.

[18] E. Gelenbe, The first decade of G-networks. Eur. J. Oper. Res. 126 (2000) 231-232.

[19] E. Gelenbe and J.M. Fourneau, Random neural networks with multiple classes of signals. Neural Comput. 11 (1999) 953-963.

[20] E. Gelenbe and J.-M. Fourneau, G-Networks with resets. Perform. Eval. 49 (2002) 179192, also in Proc. IFIP WG 7.3/ACM-SIGMETRICS Performance '02 Conf., Rome, Italy (2002).

[21] E. Gelenbe, P. Glynn and K. Sigman, Queues with negative arrivals. J. Appl. Probab. 28 (1991) 245-250.

[22] E. Gelenbe and K. Hussain, Learning in the multiple class random neural network. IEEE Trans. on Neural Networks 13 (2002) 1257-1267.

[23] E. Gelenbe and A. Labed, G-networks with multiple classes of signals and positive customers. Eur. J. Oper. Res. 108 (1998) 293-305.

[24] E. Gelenbe and I. Mitrani, Analysis and Synthesis of Computer Systems. New York, London Academic Press (1980).

[25] E. Gelenbe and G. Pujolle, Introduction to Queueing Networks. New York, Wiley (1998).

[26] E. Gelenbe and M. Schassberger, Stability of product form G-Networks. Probab. Eng. Inform. Sci. 6 (1992) 271-276.

[27] E. Gelenbe, E. Seref and Z. Xu, Simulation with learning agents. Proc. of the IEEE $\mathbf{8 9}$ (2001) 148-157.

[28] E. Gelenbe and H. Shachnai, On G-networks and resource allocation in multimedia systems. Eur. J. Oper. Res. 126 (2000) 308-318.

[29] J.R. Jackson, Networks of waiting lines. Oper. Res. 15 (1957) 234-265.

[30] J.R. Jackson, Jobshop-like queueing systems. Manage. Sci. 10 (1963) 131-142.

[31] F.P. Kelly, Reversibility and Stochastic Networks. Chichester, Wiley (1979).

$[32]$ D. Kouvatsos, Entropy maximisation and queueing network models. Ann. Oper. Res. 48 (1994) 63-126.

[33] A.V. Pechinkin and V.V. Rykov, Product form for open queueing networks with dependent service times, in Proc. Distributed Computer Communication Networks. Theory and Applications, Moscow: Institute for Information Transmission Problems RAS (1977) 171-178.

[34] M. Schwartz, Telecommunication Networks: Protocols, Modeling and Analysis. New York, Addison Wesley (1987).

[35] N.M. Van Dijk, Queueing Networks and Product Forms. New York, Wiley (1993).

[36] V.M. Vishnevskii, Theoretical Foundations of Computer Network Design. Moscow, Tekhnosfera 2003 (in Russian). 